\title{
Synchronous cecal adenocarcinoma and multiple colonic in situ carcinomas in hamartomatous polyps in a case of isolated Peutz-Jeghers syndrome
}

This article was published in the following Dove Press journal:

Gastrointestinal Cancer:Targets and Therapy

17 March 201I

Number of times this article has been viewed

\author{
Yahia Z Gad' \\ Doaa H Bakr' \\ Mohammad G El-Ebeidy ${ }^{2}$ \\ 'Department of Internal Medicine, \\ ${ }^{2}$ Department of Surgery, Mansoura \\ Specialized Medical Hospital, \\ Mansoura University, Mansoura, Egypt
}

Background: Peutz-Jeghers syndrome (PJS) is a rare autosomal dominant disease characterized by mucocutaneous pigmentation and hamartomatous polyps of the entire gastrointestinal tract. A Peutz-Jeghers polyp (PJP) in a patient without pigmentation or a family history of the disease is called an isolated or solitary PJP. Individuals with PJS carry a very high risk of developing gastrointestinal (GI) as well as extra-GI malignancies. This case report documents lesion multiplicity and their malignant potential in a young patient with PJS presenting in a serious condition for the first time.

Case report: An 18-year-old female Egyptian patient was admitted with hematochezia and remarkable anemia. After appropriate resuscitation and consent, colonoscopic evaluation revealed seven pedunculated colonic polyps at the ascending and the transverse colon, and numerous variable-sized sessile polyps were scattered all over the colon. To establish hemostasis, endoscopic polypectomy for pedunculated polyps and argon plasma photocoagulation for the bleeding sessile polyps were performed. Histopathological examination revealed cecal adenocarcinoma in one specimen and two simultaneous in situ carcinoma at the transverse and the sigmoid colon in the mucosae of the excised histologically proven hamartomatous polyps. Additionally, one focal in situ carcinoma in the resected colon was detected.

Conclusions: When considering the family history, serious GI neoplastic lesions may be unmasked in young patients with PJS who present with hematochezia, even in the absence of its characteristic mucocutaneous pigmented lesions. GI endoscopic surveillance programs should be adopted for diagnosed cases of PJS and their families. Genetic prenatal screening for early detection is the best option for primary prevention.

Keywords: isolated, Peutz-Jeghers syndrome, hematochezia

\section{Introduction}

Peutz-Jeghers syndrome (PJS) is a rare autosomal dominant disease characterized by mucocutaneous pigmentation and hamartomatous polyps of the entire gastrointestinal tract. This syndrome occurs in approximately $1 / 8300$ to $1 / 129,000$ live births. ${ }^{1}$ The typical pathological feature of Peutz-Jeghers polyp (PJP) is a smooth muscle core arising from the muscularis mucosae and ramifying into the polyp-like the branches of a tree. PJS presents with flat, pigmented, freckle-like cutaneous lesions mainly on the lower lip, perioral area, buccal mucosa, periorbital area, and the eyelids. ${ }^{2}$ A PJP in a patient without pigmentation or a family history of the disease is called an isolated or solitary PJP. ${ }^{3}$ Individuals with PJS carry a very high risk of developing gastrointestinal (GI) as well as extra-GI malignancies in the breast, ovaries, testes, and pancreas., ${ }^{4,5}$
Correspondence: Yahia Zakaria Gad Mansoura Specialized Medical Hospital, Mansoura University, Mansoura, Egypt Tel +20 I2758II 50

Fax +202230129

Emailyahiazgad@yahoo.com 
This case report documents lesion multiplicity and their malignant potential in a young patient with PJS presenting seriously for the first time.

\section{Case report}

On June 15, 2010, an 18-year-old female Egyptian patient was admitted to our hospital with hematochezia and remarkable anemia. Her past history included a tonsillectomy in 2003. Her father died in 2009 due to pulmonary embolism on the ninth postoperative day following an abdomino-perineal resection for advanced colorectal cancer. Her only sister had been investigated for three years for chronic iron deficiency anemia. On physical examination, only marked pallor was noted and no characteristic pigmentation was recognized at this time. Admission laboratory data showed a red blood cell count of 3.27 million, hemoglobin of $6.7 \mathrm{~g} / \mathrm{dL}$, hematocrit value of $22.7 \%$, and normal serum carcinoembryonic antigen level (5.0). After appropriate resuscitation and consent, an upper fiber-optic gastroscopy was performed and revealed multiple duodenal nonbleeding sessile polyps (Figure 1) and no evidence of hemobilia. On colonoscopy, seven pedunculated colonic polyps were detected at the ascending and the transverse colon and numerous variable-sized sessile polyps were scattered all over the colon. Endoscopic polypectomy was done for the large bleeding polyps. Argon plasma photocoagulation of the bleeding sessile polyps was performed until adequate hemostasis was established. Histopathologic examination revealed adenocarcinoma in one specimen in the caecum and two simultaneous in situ carcinoma at the transverse and the sigmoid colon in the mucosae of the excised histologically proven hamartomatous

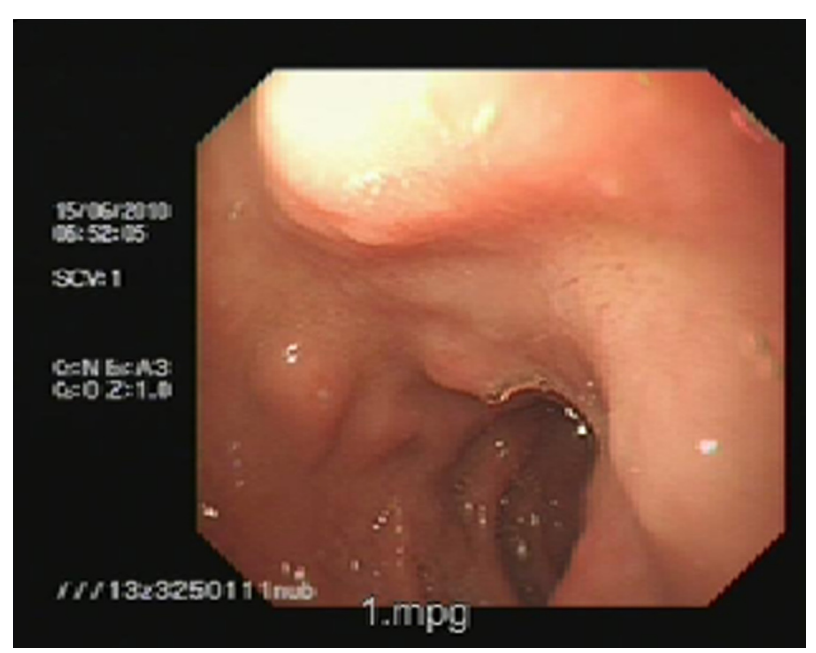

Figure I Multiple sessile duodenal polyps. polyps. The remaining polyps showed the typical appearance of PJPs with glandular disorganization, hamartomatous appearance, and ramifying branching bundles of smooth muscles (Figure 2) with no secondary malignant changes. Surgeons decided on total colectomy and end-to-side ileorectal anastomosis. At laparotomy, regional lymph nodes were excised and the small intestine was palpated thoroughly and no further lesions were detected. Examined resected specimens revealed a typical PJP with one focal in situ carcinoma in the transverse colon.

Later, full dermatological examination of the patient's body revealed no remarkable cutaneous abnormality but her sister showed melanin-pigmented spots on her lower lips (Figure 3), hands, and feet leading us to the diagnosis of PJS. HPV culturing and HIV serology were found to be negative. Genotyping regarding serine-threonine protein kinase 11 (STK 11) genomic rearrangement mutations using multiple ligation-dependent probe amplification assay has been ordered.

\section{Discussion}

PJS is a rare familial disorder with a variable penetrance, even within families; some members will manifest only hyperpigmentation, while others may manifest pigmentation and polyps. ${ }^{6}$ The melanotic macules on the skin may not develop until after the intestinal polyps appear and may fade with age. ${ }^{7,8}$ This would explain the occurrence of hamartomatous polyps in the absence of the characteristic mucocutaneous pigmented lesions in isolated PJS. A high index of suspicion is necessary to correctly diagnose this condition in light of suggestive positive family history.

Although invasive carcinoma in the small bowel has been shown to arise frequently in PJPs, ${ }^{9}$ there are few recorded

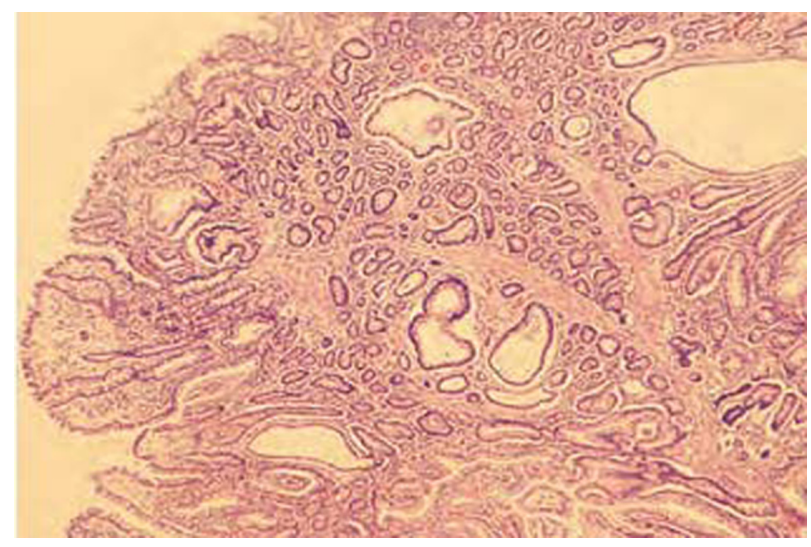

Figure 2 Typical appearance of the Peutz-Jeghers' polyp. 


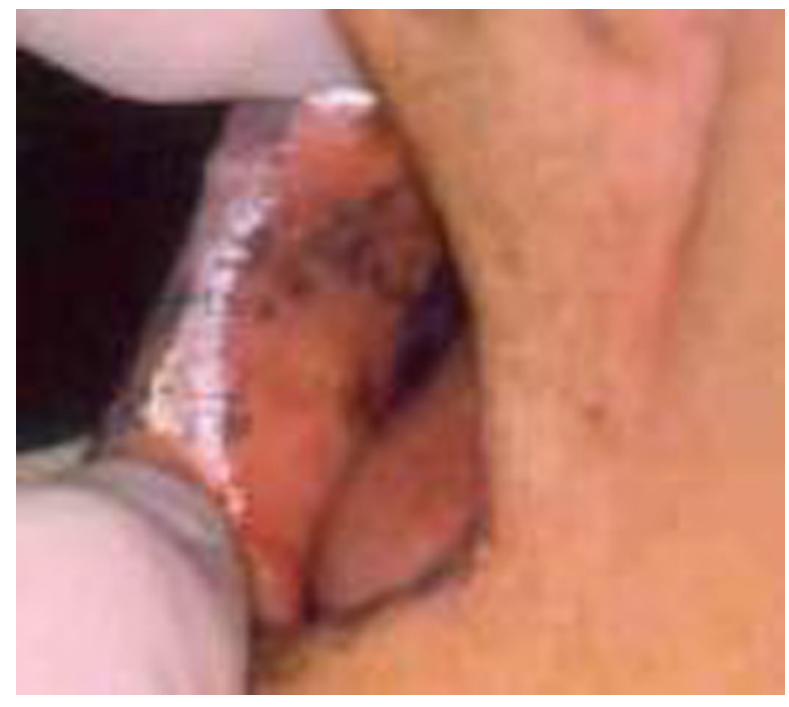

Figure 3 Melanin spots on the inner side of the lower lip of the patient's sister.

cases where a colonic carcinoma has previously shown to arise from a PJP in the colon. ${ }^{10}$ In situ carcinoma may have arisen within a hamartomatous polyp, and some of these replicating cells may have become dysplastic and then carcinomatous. Coincidental adenomatous changes may occur primarily, followed by dysplasia and carcinomatous transformation. ${ }^{11}$

In our case, it is difficult to determine whether cecal carcinoma had arisen de novo or from a PJP. But, in all detected focal colonic cancers, it is possible that they have developed as a secondary change on top of PJPs. Histopathologic examination of the other resected specimens revealed only one focal in situ carcinoma in the transverse colon; a serious finding that supported the surgeons' opinion and pointed to the malignant potential nature of PJPs.

Endoscopic polypectomy is generally useful for colonic polypoidal lesions. This was the initial option to ensure hemostasis in our case. Later on, the surgeons decided on total colectomy considering the extensive colonic involvement with polyps as well as the presence of multiple focal in situ carcinomas within the hamartomatous polyps and the pathologically proven cecal adenocarcinoma.

Compared with the standard population, PJS subjects have a relative risk of 15 for developing GI malignancies and other malignancies. Malignant tumors develop in 22\%-77\% of PJS patients, and $27 \%-59 \%$ of these cancers are located in the GI tract where most are in the colon followed by the stomach and the small intestine. ${ }^{12}$ Thus, colonic cancer could be anticipated in a young patient with PJS as there is a generalized increased cancer risk in this condition. It becomes a strong obligation for those patients and their first-degree relatives to undergo regular surveillance endoscopic examination of the GI tract every $3-5$ years for early cancer detection as previously recommended. ${ }^{13}$

\section{Conclusion}

Hematochezia is a serious emergency. When considering family history, serious GI neoplastic lesions may be unmasked in young patients with PJS who present with hematochezia even in the absence of its characteristic mucocutaneous pigmented lesions. GI endoscopic surveillance programs should be adopted for diagnosed cases of PJS and their families. Genetic prenatal screening for early detection of such cases is the best option for primary prevention when adequate resources are available.

\section{Learning points}

1. Subjects with PJS are not necessarily identified by their characteristic mucocutaneous pigmentation.

2. Those without cutaneous lesions are at substantial risk of developing GI and/or extra-GI malignancies.

3. Those subjects and their first-degree relatives should be scheduled in surveillance endoscopic programs.

4. Mucocutaneous pigmentation of PJS could be easily missed and should be carefully inspected in non fairskinned subjects.

\section{Acknowledgments}

Many thanks to the patient and her sister for their cooperation. Special thanks to the pathology department staff for their valuable help throughout the work.

\section{Disclosure}

No conflicts of interest were declared in relation to this paper.

\section{References}

1. Finan MC, Ray MK. Gastrointestinal polyposis syndromes. Dermatol Clin. 1989;116:419-434.

2. Bhattacharya S, Mahapatra SR, Nangalia R, et al. Melaena with PeutzJeghers syndrome: a case report. J Medical Case Reports. 2010;4:44.

3. Stone Y, Nakano S, Takeda I, Kumada T, Kiriyama S, Hisanaga Y. Solitary hamartomatous polyp of Peutz-Jeghers type in the jejunum resected endoscopically. Gastrointest Endosc. 2000;51:620-622.

4. Buck JL, Hamed RK, Lichtenstein JE, Sobin LH. Peutz-Jeghers syndrome. Radiographics. 1992;12:365-378.

5. Glardiello FM, Welch SB, Hamilton SR, et al. Increased incidence of cancers in the Peutz-Jeghers syndrome. N Engl J Med. 1987;316: 1511-1514

6. Kobacova M, Rejchrt S, Bures J. Peutz-Jeghers syndrome: diagnostic and therapeutic approach. World J Gastroenterol. 2009;15:5397-5408. 
7. Bartholomew LG, Moore CE, Dahlin DC, Waugh LM. Intestinal polyposis associated with mucocutaneous pigmentation. Surg Gynaecol Obstet. 1962;115:1-15.

8. Burt RW, Berenson MM, Lee RG, Tolman KG, Preston JW, Gardener EG. Upper gastrointestinal polyposis. Gastroenterology. 1984;86:295-301.

9. Matuchansky C, Babin P, Coutrot S, Druart F, et al. Peutz-Jeghers syndrome with matastasising carcinoma arising from a jejunal hamartoma. Gastroenterology. 1979;77:1311-1315.

10. Miller LJ, Bartholomew LG, Dozois RR, Dahlin DC. Adenocarcinoma of the rectum arising from a hamartomatous polyp in a patient with Peutz-Jeghers syndrome. Dig Dis Sci. 1983;28:1047-1051.
11. Perzin KH, Bridge MF. Adenocarcinoma and carcinomatous changes in hamartomatous polyps of the small intestine (Peutz-Jeghers syndrome): report of a case and review of the literature. Cancer. 1982;49: 971-983.

12. Mullhaupt B, Bauerefeind P, Kurrer MO, Fried M. Anal squamous cell carcinoma in a patient with Peutz-Jeghers' syndrome. Dig Dis Sci. 2001;46:273-277.

13. Spigejman AD, Arese P, Philips RK. Polyposis. The Peutz-Jeghers' syndrome. Br J Surg. 1995;82:1311-1314.

\section{Publish your work in this journal}

Gastrointestinal Cancer: Targets and Therapy is an international, peer-reviewed, open access journal focusing on gastro-intestinal cancer research, identification of therapeutic targets and the optimal use of preventative and integrated treatment interventions to achieve improved outcomes, enhanced survival and quality of life for the cancer patient. The manuscript management system is completely online and includes a very quick and fair peer-review system. Visit http://www.dovepress.com/testimonials.php to read real quotes from published authors.

Submit your manuscript here: http://www.dovepress.com/gastro-intestinal-cancer-targets-and-therapy-journal 